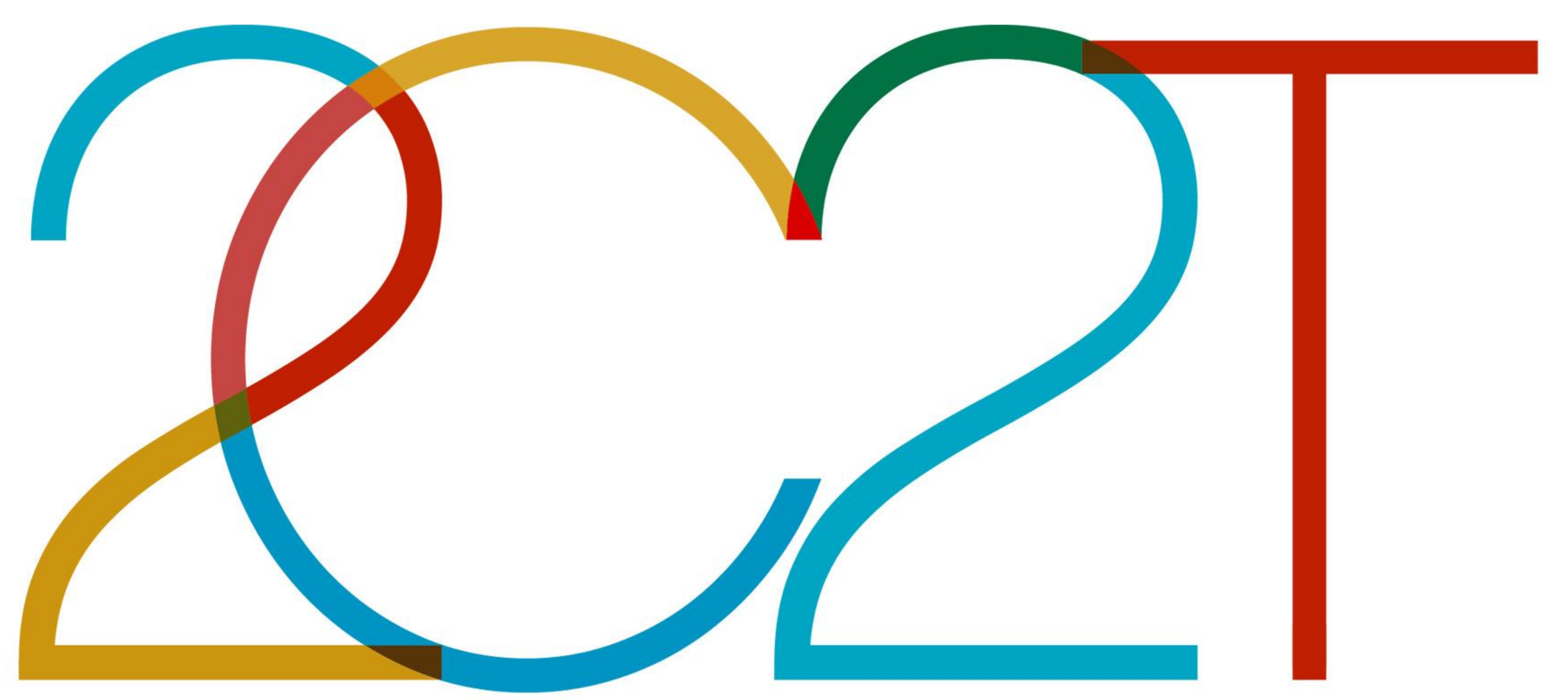

\section{CENTRO DE CIÊNCIAE TECNOLOGIATÊXTIL}

\author{
www.2c2t.uminho.pt
}

\title{
Development of biodegradable electrospun dressing based on PVA/CA with antimicrobial potential for chronic wounds
}

Marta A. Teixeira*; Tânia D. Tavares, Ana R. M. Ribeiro, M. Teresa P. Amorim and Helena P. Felgueiras Centro de Ciência e Tecnologia Têxtil (2C2T), Universidade do Minho, Portugal *martaalbertinateixeira@gmail.com

Introduction

The incidence of chronic wounds (CW) is growing at an accelerated rate around the world. CW are unable to follow the normal healing steps, stalling at a self-perpetuating inflammatory stage. Pressure, excessive proteases and inappropriate treatment are some of the factors that hinder the healing process, but the main cause of the problem remains the increased bacterial load [1]

To address this problem, wound dressings with a new intricated nanoarchitecture and functionalized with active biomolecules are being developed to treat $\mathrm{CW}$. The introduction of antimicrobial peptides (AMPs), as pexiganan (or MSI-78), which display a broad spectrum of antibacterial activity against both Gram-negative and Gram-positive and reduce bacterial resistance, has been considered a viable solution [2].

Our main goal focuses on the development, through electrospinning technique, of a biodegradable and 3D nano-structured dressing based on poly(vinyl alcohol) (PVA) and cellulose acetate (CA), functionalized with pexiganan, as potential antimicrobial agent.

\section{Electrospun mats production}

PVA (88\% hydrolyzed and Mw 78,000) was purchased from Polysciences, Warrington, USA. CA (Mw 30,000) was acquired from Sigma Aldrich, USA. The acetic acid (glacial) $100 \%$ was purchased from Merck, Darmstadt, Germany. Glutaraldehyde (GA, 25\% aqueous solution) was obtained from Sigma Aldrich.

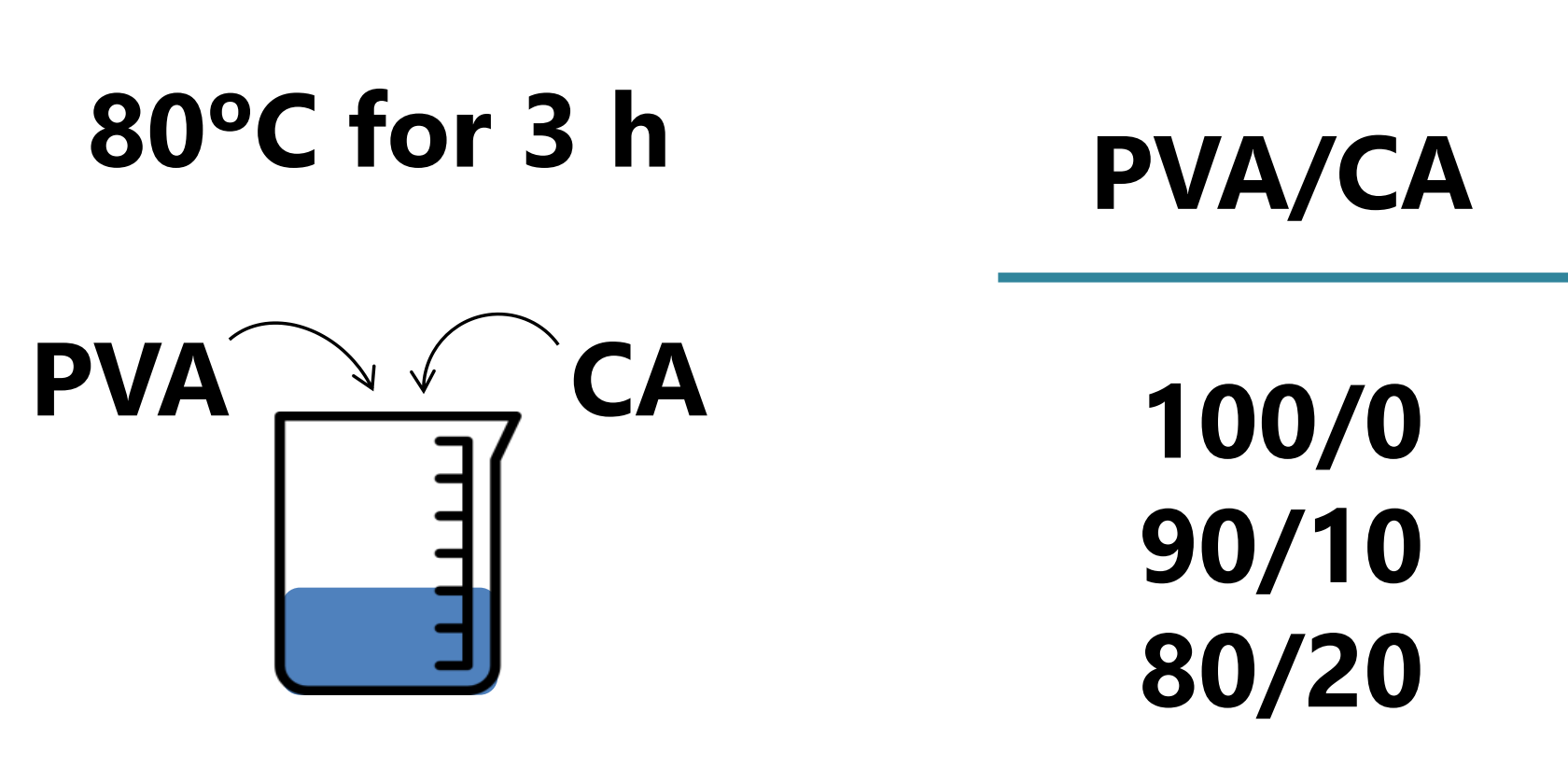

Polymeric solutions:

Concentration: $10 \%(w / v)$ in $75 / 25 \%(v / v)$ acetic acid/ $/ \mathrm{dH}_{2} \mathrm{O}$

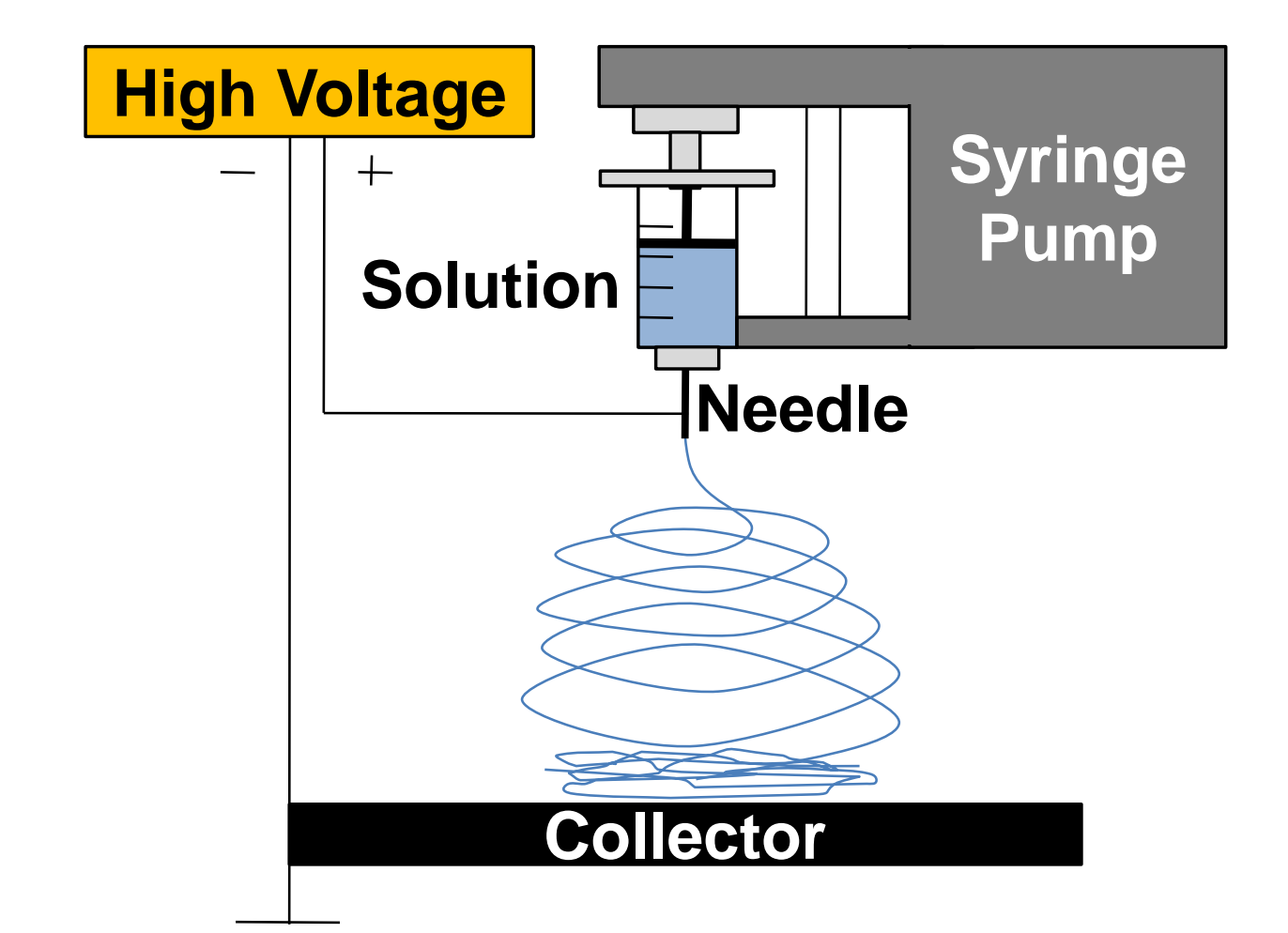

Electrospinning process

\begin{tabular}{|c|}
\hline Parameters applied: \\
Voltage: $\mathbf{2 5} \mathbf{~ k V} ;$ \\
Feed rate: $\mathbf{0 . 8} \mathbf{~ m L / h ;}$ \\
Distance between syringe \\
and collector: $\mathbf{1 8} \mathbf{~ c m}$. \\
\hline
\end{tabular}

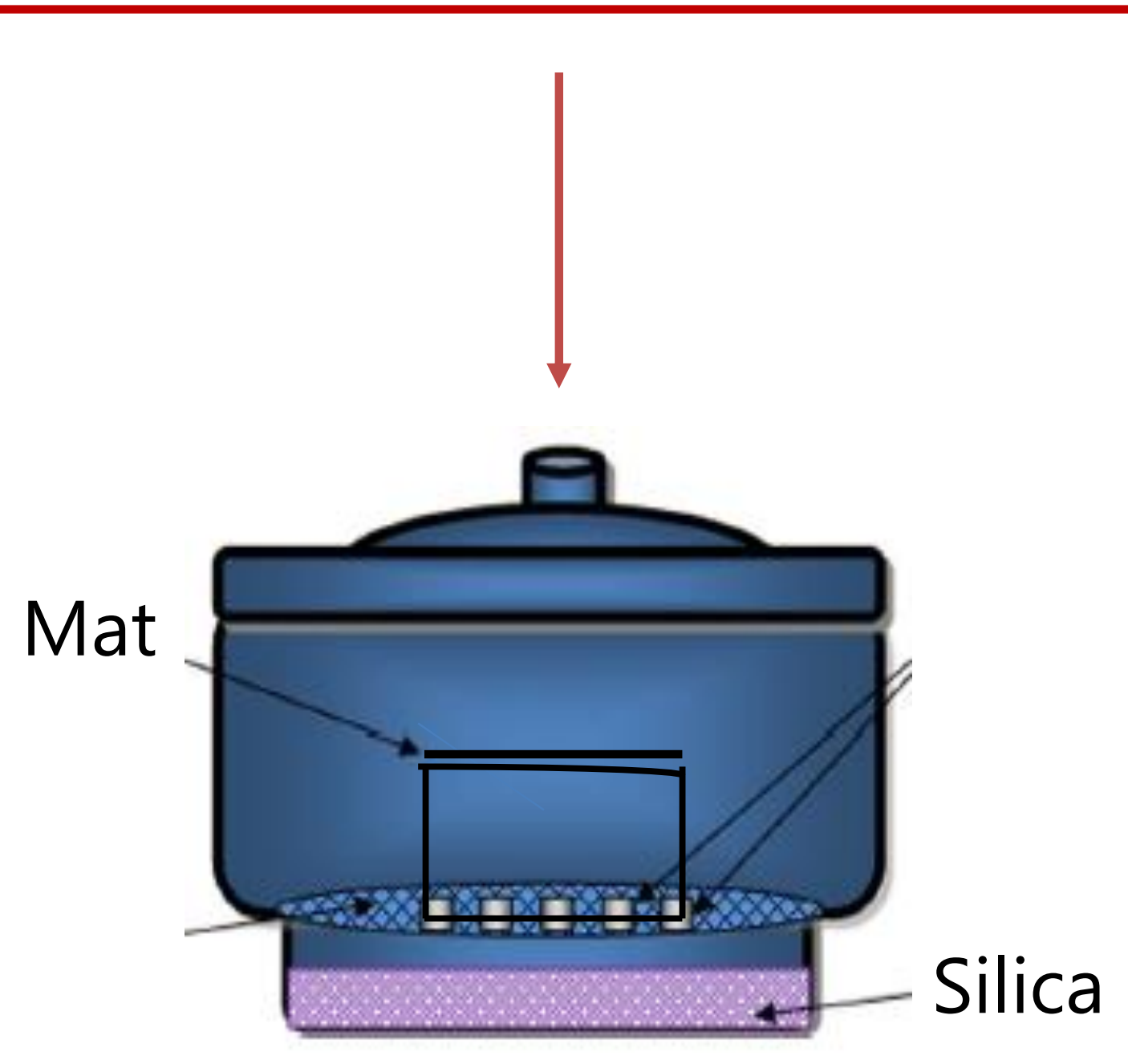

Crosslinking process

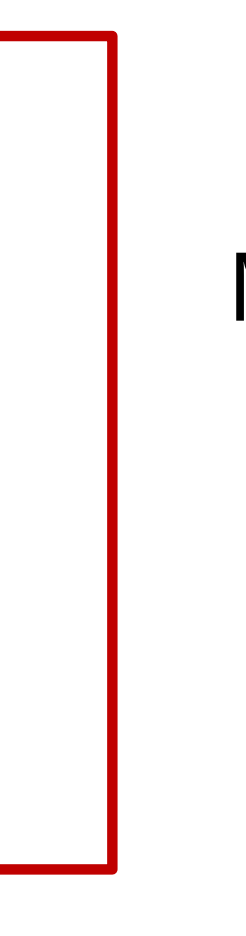

\section{Characterization}

\section{Morphological evaluations of the produced nanofibers}
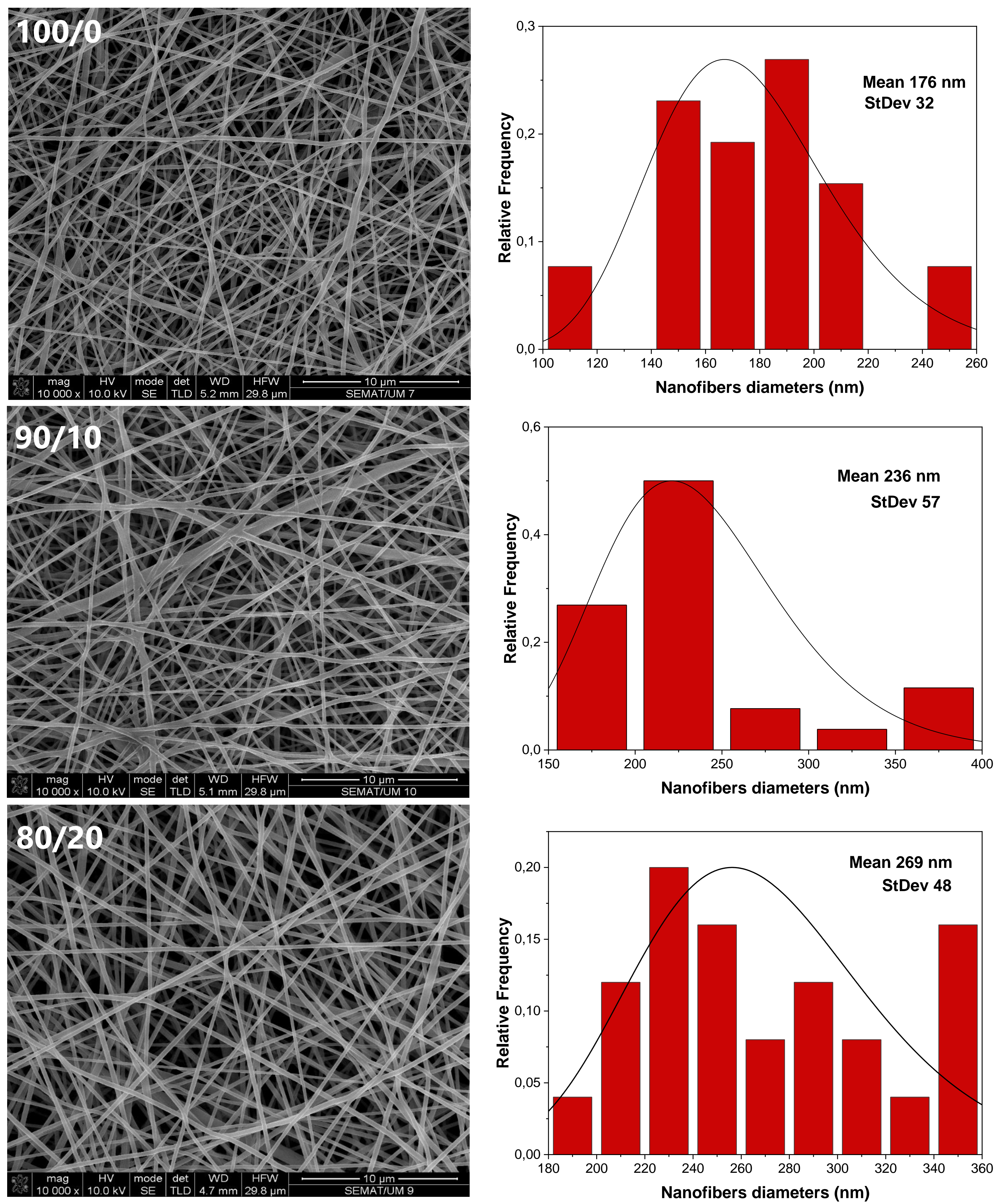

Figure 1. FEG-SEM micrographs and diameter distribution of 100/0, 90/10, $80 / 20$ PVA/CA mats crosslinked with glutaraldehyde $(n=50)$. Formation of uniform and bead-free fibers.

\section{Functionalization of electrospun mats with Pexiganan}

"Surface Initiated": N-terminal cysteine-modified Pexignan is immobilized on the surface of electrospun mats by succinimidyl-ester (NHS)-PEG2-maleimide.

\section{Temperature: $60^{\circ} \mathrm{C}$}

Glutaraldehyde exposure: $7 \mathrm{~h}$

Amount of crosslinker: $6 \mathrm{ml}$

Concentration: $2,56 \mathrm{M}$

Drying temperature: $\mathrm{RT}$ in a

controlled desiccator

\section{Molecules}

Covalent binding

\section{in Solution $\bullet$ - \\ Initiator $\uparrow \uparrow \uparrow \uparrow \uparrow \uparrow \uparrow \uparrow \uparrow \uparrow$ \\ Conclusions \\ Preliminary data demonstrated Pexiganan activity against Staphylococcus aureus, Staphylococcus epidermidis, Pseudomonas aeruginosa and Escherichia coli with values of minimum inhibitory concentrations (MIC) ranging from 7.8 to $62.5 \mu \mathrm{g} / \mathrm{mL}$.}

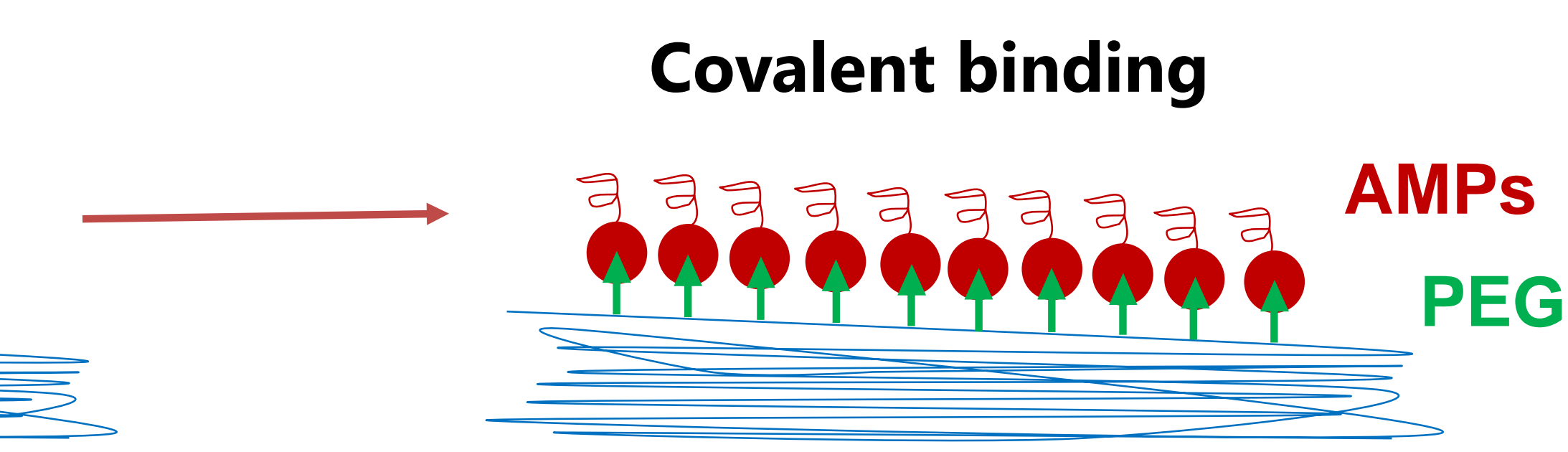

References: [1] Felgueiras H.P. et al., Appl. Polym., 137, 2020; [2] Felgueiras H.P. et al., Colloids Surf. B, 156:133, 2017.

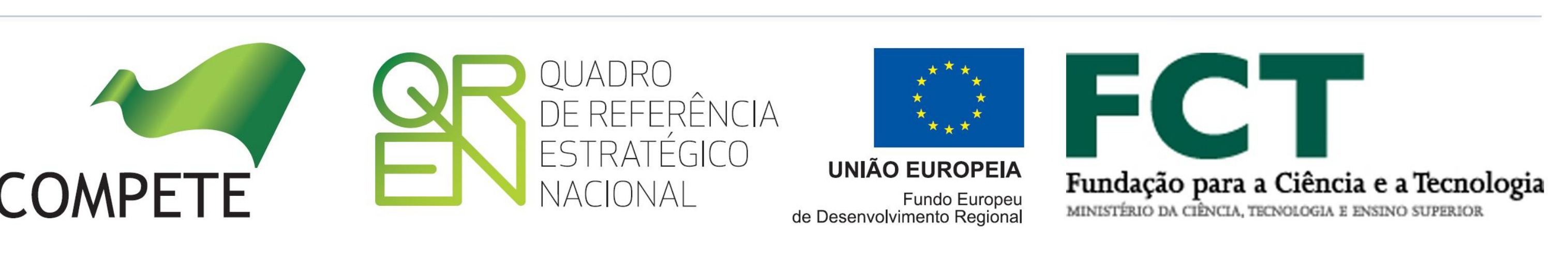

